\title{
Patients Evaluate Visit Notes Written by Their Clinicians: a Mixed Methods Investigation
}

\author{
Suzanne G. Leveille, RN, PhD 1,2,3, Patricia Fitzgerald, MSC ${ }^{2}$, Kendall Harcourt, MPA ${ }^{2}$, \\ Zhiyong Dong, $\mathrm{MSC}^{2}$, Sigall Bell, MD',3, Steve O'Neill, JD, MSW',3, \\ Catherine DesRoches, DrPH 2,3, Leonor Fernandez, MD 2,3, Sara L. Jackson, MD, MPH', \\ Jared W. Klein, MD, MPH ${ }^{4}$, Rebecca Stametz, MPH, DEd ${ }^{5}$, Tom Delbanco, $M D^{2,3}$, and \\ Jan Walker, RN, MBA ${ }^{2,3}$
}

\begin{abstract}
${ }^{1}$ College of Nursing and Health Sciences, University of Massachusetts, Boston, MA, USA; ${ }^{2}$ Division of General Medicine, Beth Israel Deaconess Medical Center, Boston, MA, USA; ${ }^{3}$ Harvard Medical School, Boston, MA, USA; ${ }^{4}$ Division of General Internal Medicine, School of Medicine, University of Washington, Seattle, WA, USA; ${ }^{5}$ Steele Institute of Health Innovation, Geisinger, Danville, PA, USA.
\end{abstract}

\begin{abstract}
BACKGROUND: Patients actively involved in their care demonstrate better health outcomes. Using secure internet portals, clinicians are increasingly offering patients access to their narrative visit notes (open notes), but we know little about their understanding of notes written by clinicians.
\end{abstract}

OBJECTIVE: We examined patients' views on the clarity, accuracy, and thoroughness of notes, their suggestions for improvement, and associations between their perceptions and willingness to recommend clinicians to others. DESIGN: We conducted an online survey of patients in 3 large health systems, June-October 2017. We performed a mixed methods analysis of survey responses regarding a self-selected note.

PARTICIPANTS: Respondents were 21,664 patients aged 18 years or older who had read at least 1 open note in the previous 12 months.

MAIN MEASURES: We asked to what degree the patient recalled understanding the note, whether it described the visit accurately, whether anything important was missing, for suggestions to improve the note, and whether they would recommend the authoring clinician to others.

KEY RESULTS: Nearly all patients (96\%) reported they understood all or nearly all of the self-selected note, with few differences by clinician type or specialty. Overall, 93\% agreed or somewhat agreed the note accurately described the visit, and 6\% reported something important missing from the note. The most common suggestions for improvement related to structure and content, jargon, and accuracy. Patients who reported understanding only some or very little of the note, or found inaccuracies or omissions, were much less likely to recommend the clinician to family and friends.

CONCLUSIONS: Patients overwhelmingly report understanding their visit notes and usually find them accurate, with few disparities according to sociodemographic or health characteristics. They have many suggestions for improving their quality, and if they understand a note

Received February 3, 2020

Accepted June 25, 2020

Published online July 15, 2020 poorly or find inaccuracies, they often have less confidence in their clinicians.

$\mathrm{J}$ Gen Intern Med 35(12):3510-6 DOI: $10.1007 / \mathrm{s} 11606-020-06014-7$

(c) Society of General Internal Medicine 2020

\section{INTRODUCTION}

Engaged patients have better health outcomes, ${ }^{1}$ and clinicians strive to involve patients more actively in their care. Today, many patients access their health information via secure, electronic patient portals. ${ }^{2}$ Increasingly, this access includes the narrative visit notes clinicians prepare, referred to as open notes. ${ }^{3}$

Patients report many benefits from reading open notes, including feeling more in control of their care, helping them adhere to their medication regimens, and enabling them to understand their health conditions and care plans better. ${ }^{4-9}$ However, we know little of their perspectives on notes written by different clinicians in different specialties, or of their thoughts on how notes might be changed to meet their needs more effectively.

As patients have become more experienced, we sought to understand their perspectives about reading a note from a single encounter with a clinician from any specialty. We wanted to understand their views on its clarity, accuracy, and completeness, and their suggestions for improving the note. Based on our previous work, we hypothesized that patients with less education would report more difficulty understanding the notes, especially those written by physicians compared with those written by other health professionals. ${ }^{4,5} \mathrm{We}$ anticipated that notes prepared by primary care clinicians would be understood more readily than those written by specialists. We expected that about a quarter of patients would find the note inaccurate, and that about a tenth would report important information missing. ${ }^{5,10}$ And we hypothesized that patients' perspectives about their notes would be associated with their willingness to recommend their clinicians to others. 


\section{METHODS}

We surveyed adult outpatients about reading their clinicians' visit notes through online portals affiliated with 3 US health systems: Beth Israel Deaconess Medical Center (BIDMC) in Boston, Geisinger health system in Pennsylvania, and University of Washington Medicine (UW) in Seattle. We conducted the survey online, June-October 2017, with patients $\geq 18$ years of age who had $\geq 1$ office visit and had logged into the patient portal at least once in the previous 12 months. To enhance our response rate, we sent patients reminder messages and offered financial incentives through a lottery. ${ }^{11}$ Of 136,815 invited to participate, 29,656 (21.68\%) responded; 22,947 were patients who had opened at least 1 note in the previous 12 months, verified by portal tracking data. Knowing that patients sometimes confuse notes with the portal itself, with other parts of the record, or with an "after visit summary," we described notes in both the survey invitation and in a question, and we showed a screenshot of the location of notes on their portal. Detailed survey methods have been published. ${ }^{5}$

We considered asking patients to refer to the most recent note but decided instead to have them choose a note that they recalled: "Please think about one visit note written by a doctor, nurse practitioner, physician assistant, or mental health professional." We asked them to consider notes by these clinicians because we wanted to know about notes that were likely part of an ongoing relationship, rather than a single visit encounter. We asked patients to identify the type of clinician who wrote the note they would be describing. At BIDMC and Geisinger, patients reported the specialty of the clinical office for the note they selected; UW patients were not asked this question.

We asked 4 closed-ended questions: (1) Who wrote the note (type of clinician, do not know)? (2) How much of the note did you understand (very little, some, almost everything, everything)? (3) The note accurately described the visit (agree, somewhat agree, somewhat disagree, disagree, do not know/ not sure), and (4) Was anything important missing from the note (yes, no, do not know/not sure)? If patients indicated they did not understand much of the note, we asked where they looked for help. We asked them to write free text describing suggestions about how the note could have been better. Finally, we asked whether they would recommend the clinician to other family members or friends. The survey instrument is available on request.

\section{Data Analysis}

We used descriptive statistics to summarize responses and chisquare tests to evaluate differences between groups. In the clinician type analysis, we excluded notes by mental health professionals because they comprised $<1 \%$ of responses. We limited the analysis of differences among primary care, combined specialties, and individual specialty practices to categories with $\geq 150$ observations. Finally, we examined patients' evaluations of their notes by whether they would recommend their clinicians to others.
We previously compared respondents with nonrespondents according to sociodemographic characteristics (age, sex, race, and insurance status). ${ }^{5}$ In this study, we also used diagnosis codes from administrative data to compare them according to the Charlson Comorbidity Index, ${ }^{12}$ and prevalence of 2 major chronic diseases: coronary heart disease $^{13}$ (ICD-10 codes I20-I25) and diabetes ${ }^{14}$ (types 1 and 2, ICD-10 codes E10-E14).

We completed a thematic analysis to evaluate 4562 free-text responses to the question, "Do you have suggestions about how this visit note could have been better?" "I5 In 4 iterative steps, we defined, revised, and finalized 9 thematic and 2 "other" coding categories, and we decided to exclude responses with fewer than 5 words. Two coders then reviewed and coded the remaining responses, assigning up to 3 codes per response. We calculated the Cohen's kappa statistic and the proportion of responses in which there was complete agreement. After analysis of the agreement rates, we decided to present responses in which there was complete agreement. Finally, the 2 coders reviewed the comments in each category to select representative examples.

\section{RESULTS}

\section{Quantitative Analysis}

A total of 21,664 patients (the $94 \%$ of patient respondents who read at least 1 note) reported on their experiences in reading individual notes; $63 \%$ were women, $75 \%$ were aged 45 years or older, and $85 \%$ were white. The majority ( $72 \%$ ) had completed college, $57 \%$ reported being employed, and $85 \%$ reported good or excellent health. Compared with non-respondents, respondents had higher scores on the Charlson Index, with $24.6 \%$ having scores of 1 or higher versus $18.1 \%$ among non-respondents, and higher prevalence of diabetes $(7.7 \%$ vs $6.2 \%)$ and coronary heart disease (3.5\% vs $2.2 \%)$.

Overall, $96 \%$ of patients, and at least $91 \%$ of patients in all demographic and health categories, reported they understood all or nearly all of their self-selected notes (Table 1). We observed a 5\% difference in proportions who understood their notes among those who completed college, compared to those with high school or less education ( $97 \%$ and $92 \%$, respectively). Most respondents (93\%) found that the note accurately described the visit, and few, $6 \%$ overall, reported something important to them missing from the note. Patients reporting fair or poor health were least likely to agree that the note was accurate ( $88.6 \%$ vs $94.4 \%$ of those in better health) and most likely to report something important was missing $(10.5 \%$ vs $4.9 \%$ of those in better health).

We found no material differences in the proportions of patients who understood the note according to type of clinician, with $97 \%$ reporting they understood all or most of notes written by physicians or NPs, and $96 \%$ reported understanding notes written by PAs (Table 2). Among the $4 \%$ of patients who reported understanding some or little of the note, most looked 
Table 1 Responses from 21,664 Patients About an Individual Note, According to Patient Characteristics

\begin{tabular}{|c|c|c|c|c|}
\hline \multirow[t]{2}{*}{ Patient characteristic } & \multirow{2}{*}{$\begin{array}{l}\text { Understood everything } \\
\text { or almost everything } \\
\text { in the note } \\
n(\%)\end{array}$} & \multirow{2}{*}{$\begin{array}{l}\text { Agree or somewhat agree } \\
\text { that note accurately } \\
\text { described the visit } \\
n(\%)\end{array}$} & \multirow{2}{*}{$\begin{array}{l}\text { Something important } \\
\text { was missing from } \\
\text { the note } \\
n(\%)\end{array}$} & \multirow{2}{*}{$\begin{array}{l}\text { Definitely or probably } \\
\text { would recommend } \\
\text { the clinician } \\
n(\%)\end{array}$} \\
\hline & & & & \\
\hline Total & $20,813(96.07)$ & $20,218(93.35)$ & $1251(5.78)$ & $18,554(92.11)$ \\
\hline \multicolumn{5}{|l|}{$\mathrm{Age}^{\mathrm{a}}$} \\
\hline $18-24$ & $685(96.34)$ & $681(95.78)$ & $32(4.50)$ & $581(89.25)$ \\
\hline $25-44$ & $4567(96.49)$ & $4444(93.89)$ & $251(5.30)$ & $4051(90.67)$ \\
\hline $45-64$ & 8709 (96.22) & $8389(92.71)$ & $563(6.22)$ & $7766(91.72)$ \\
\hline $65+$ & $6852(95.58)^{*}$ & $6704(93.57)^{* * *}$ & $405(5.65)^{*}$ & $6156(93.88)^{* * * *}$ \\
\hline \multicolumn{5}{|l|}{$\mathrm{Sex}^{\mathrm{a}}$} \\
\hline Female & $13,178(96.43)$ & $12,601(92.24)$ & $896(6.56)$ & $11,515(90.83)$ \\
\hline Male & $7635(95.46)^{* *}$ & $7617(95.25)^{* * *}$ & $355(4.44)^{* * * *}$ & $7039(94.28)^{* * * *}$ \\
\hline \multicolumn{5}{|l|}{ Race } \\
\hline White & $17,363(96.40)$ & $16,906(93.86)$ & $968(5.37)$ & $11,561(92.65)$ \\
\hline Black & $514(94.66)$ & $513(94.48)$ & $24(4.42)$ & $473(92.56)$ \\
\hline Asian & $1016(94.42)$ & $1026(95.35)$ & $55(5.11)$ & $911(92.77)$ \\
\hline Other & $666(94.60)$ & $616(87.50)$ & $74(10.51)$ & $553(86.14)$ \\
\hline Multiple races & $724(94.76) * * *$ & $679(88.87)^{* * *}$ & $76(9.95)^{* * *}$ & $607(87.46)^{* * *}$ \\
\hline \multicolumn{5}{|l|}{ Ethnicity } \\
\hline Non-Hispanic & $19,634(96.14)$ & $19,114(93.59)$ & $1164(5.70)$ & $17,585(92.26)$ \\
\hline Hispanic & $743(95.50)$ & $713(91.65)$ & $48(6.17)$ & $646(90.86)^{*}$ \\
\hline \multicolumn{5}{|l|}{ Education } \\
\hline Masters/doctoral degree & $7748(96.74)$ & 7488 (93.49) & $524(6.54)$ & $7058(92.65)$ \\
\hline 4-year degree/some grad school & $7045(96.45)$ & $6894(94.39)$ & $380(5.20)$ & $6316(92.03)$ \\
\hline Some college/technical school & $4504(95.83)$ & $4349(92.53)$ & $278(5.91)$ & $3910(91.48)$ \\
\hline High school or less & $1281(91.76)^{* * *}$ & $1282(91.83)^{* * *}$ & $48(3.44)^{* * *}$ & $1117(92.31)$ \\
\hline \multicolumn{5}{|l|}{ Employment } \\
\hline Employed/self-employed & $11,805(96.65)$ & $11,511(94.24)$ & $608(4.98)$ & $10,595(91.94)$ \\
\hline Retired & $6423(95.69)$ & $6289(93.70)$ & $370(5.51)$ & $5768(93.64)$ \\
\hline Not employed ${ }^{b}$ & $2283(94.69) * * *$ & $2156(89.42)^{* * *}$ & $240(9.95) * * *$ & $1984(89.53) * * *$ \\
\hline \multicolumn{5}{|l|}{ General health } \\
\hline Excellent/very good/good & $17,433(96.58)$ & $17,037(94.38)$ & $882(4.89)$ & $15,722(92.98)$ \\
\hline Fair/poor & $3092(93.58)^{* * *}$ & $2927(88.59)^{* * *}$ & $346(10.47)^{* * *}$ & $2631(87.61)^{* * *}$ \\
\hline \multicolumn{5}{|l|}{ Primary language } \\
\hline English & $18,890(96.24)$ & $18,356(93.51)$ & $1118(5.70)$ & $16,912(92.25)$ \\
\hline Other & $1524(94.42)^{*}$ & $1508(93.43)$ & $96(5.95)^{* * * *}$ & $1349(91.27)$ \\
\hline
\end{tabular}

${ }^{a}$ Age and sex information from administrative databases

${ }^{b}$ Not employed includes homemakers, unemployed, and disabled

Chi-square test for overall differences between groups, $p$ value $<0.05^{*},<0.01 * *,<0.001^{* * *}$

for help: $35 \%$ searched the internet, $27 \%$ asked a clinician, $7 \%$ asked a friend or family member, and $27 \%$ reported not looking for help (data not shown).

Referring to notes written by physicians, $95 \%$ of patients reported that the note accurately described the visit, compared with $92 \%$ of notes by NPs, and $90 \%$ by PAs. Fewer than $10 \%$ of patients overall reported something important to them missing from the note: $5 \%$ of physicians' notes, $6 \%$ of NPs' note, and $9 \%$ of PAs' notes. Nearly all patients (93\%) who reported on notes by physicians said they would definitely or probably recommend the clinician to family or friends, compared with $89 \%$ of NPs and $85 \%$ of PAs.

There were few differences according to clinician specialty (Table 2). Ninety-seven percent of patients reporting on primary care notes, and $94 \%$ of those reporting on specialty notes, understood all or most of the notes, and $89 \%$ to $96 \%$ felt they were accurate. Across specialties, 3-9\% reported something important missing from the note. In the different specialties, $88-96 \%$ of patients would recommend the clinician to others.

We examined the patients' likelihood for recommending the authoring clinician to family or friends according to how they responded to the 3 questions about note content
(Fig. 1). Among patients who understood their note, $93 \%$ would recommend their clinician to others, but this proportion dropped to $77 \%$ among those who understood some or very little of their note. While only $4 \%$ of patients reported that the note did not accurately describe the visit, they were much less likely to recommend their provider to others, compared with those finding the note accurate $(43 \%$ and $95 \%$, respectively). Similarly, patients who found something important missing were less likely to recommend their provider, compared with those not finding anything important missing (54\% and $96 \%$, respectively).

\section{Qualitative Analysis}

When asked how the note could have been better, 4562 (21.1\%) respondents offered comments. After excluding 750 responses containing fewer than 5 words, we coded the remaining 3812 comments. The 2 coders had fair to good agreement based on all permutations of 11 categories, with kappa $=0.56,{ }^{16}$ and they had full agreement on up to 3 coded categories in 2376 responses (62.3\%) reported here (Table 3). 
Table 2 Patient Responses About a Note Authored by Different Types of Clinicians and in Offices of Different Specialties

\begin{tabular}{|c|c|c|c|c|c|}
\hline & Total & $\begin{array}{l}\text { Understood } \\
\text { everything } \\
\text { or almost } \\
\text { everything } \\
\text { in the note }\end{array}$ & $\begin{array}{l}\text { Agree or somewhat } \\
\text { agree that note accurately } \\
\text { described visit }\end{array}$ & $\begin{array}{l}\text { Something } \\
\text { important } \\
\text { was missing } \\
\text { from } \\
\text { the note }\end{array}$ & $\begin{array}{l}\text { Definitely or } \\
\text { probably } \\
\text { would } \\
\text { recommend } \\
\text { the clinician }\end{array}$ \\
\hline & $N(\%)$ & $N(\%)$ & $N(\%)$ & $N(\%)$ & $N(\%)$ \\
\hline \multicolumn{6}{|l|}{ Note author ${ }^{\mathrm{a}}$} \\
\hline Doctor & $17,475(81.42)$ & $16,926(96.88)$ & $16,551(94.74)$ & $901(5.16)$ & $16,208(92.94)$ \\
\hline Nurse practitioner & $1682(7.84)$ & $1627(96.73)$ & $1553(92.39)^{* * *}$ & $104(6.19)^{*}$ & $1500(89.39)^{* * *}$ \\
\hline Physician assistant & $822(3.83)$ & $787(95.74)$ & $736(89.54)^{* * *}$ & $75(9.12)^{*}$ & $700(85.37)^{* * *}$ \\
\hline Do not know/not sure & $1485(6.92)$ & $1274(86.31)^{* * *}$ & $1203(81.56)^{* * *}$ & $134(9.07) * * *$ & $1281(87.5)$ \\
\hline Total notes by these authors & $21,464(100)$ & $20,614(96.10)$ & $20,043(93.45)$ & $1214(5.66)$ & $18,408(92.33)$ \\
\hline \multicolumn{6}{|l|}{ All offices ${ }^{\mathrm{b}}$} \\
\hline Primary care & $7083(57.06)$ & $6899(97.40)$ & $6732(95.07)$ & $270(3.81)$ & $6297(93.59)$ \\
\hline Combined specialties & $5330(42.94)$ & $5029(94.35) * * *$ & $4907(92.06)^{* * *}$ & $361(6.77) * * *$ & $4522(91.13)^{* * *}$ \\
\hline \multicolumn{6}{|c|}{ Specialty offices with $\geq 150$ observations } \\
\hline Gastroenterology & 642 & $601(94.50)^{* * *}$ & $590(92.77)^{*}$ & $51(8.02)^{* * * *}$ & $542(90.94)^{*}$ \\
\hline $\mathrm{Ob} / \mathrm{Gyn} /$ women's health & 485 & $458(94.43) * * *$ & $453(93.40)$ & $32(6.60)^{* * *}$ & $411(91.13)^{*}$ \\
\hline Neurology/neurosurgery & 483 & $446(92.34) * * *$ & $434(89.86) * * *$ & $37(7.66) * * *$ & $404(88.99)^{* * *}$ \\
\hline Oncology/hematology/cancer & 473 & $449(94.93) * * *$ & $454(95.98)$ & $15(3.17)$ & $436(95.61)$ \\
\hline Orthopedics & 440 & $414(94.09) * * *$ & $393(89.32)^{* * * *}$ & $40(9.09)^{* * *}$ & $369(90.00)^{* * *}$ \\
\hline Heart and vascular care & 434 & $398(91.71) * * *$ & $404(93.09)$ & $22(5.07)$ & $385(94.83)$ \\
\hline Endocrinology & 356 & $348(97.75)$ & $335(94.10)$ & $16(4.49)$ & $320(90.91)$ \\
\hline Dermatology & 337 & $321(95.25)^{*}$ & $307(91.10)^{* * *}$ & $22(6.53)^{*}$ & $276(88.18)^{* *}$ \\
\hline Surgery & 227 & $261(95.26) *$ & $248(90.51) * * *$ & $22(8.03) * *$ & $225(91.46)$ \\
\hline Rheumatology & 233 & $233(95.88)$ & $223(91.77)$ & $16(6.58)$ & $206(88.41)^{* * *}$ \\
\hline
\end{tabular}

${ }^{a}$ Excludes 200 responses about notes by mental health professionals due to small sample size

${ }^{b}$ Data from BIDMC and Geisinger. UW survey did not ask for office specialty

$P$ values for chi-square test compared with primary care, $p$ value $<0.05^{*},<0.01 * *,<0.001 * * *$

The most common suggestions related to the structure and content of notes. Patients wanted new information prominently featured at the top of the note, including clear instructions about next steps, instructions about referrals, and explanations of test results. They felt that notes were not always sufficiently personalized or detailed, which they often attributed to copying old information and using templates. Comments about use of medical jargon were almost as frequent, with many respondents suggesting technical solutions such as links to glossaries.
Patients also wrote about the accuracy and completeness of notes, particularly mistakes in documenting the visit discussion and outdated patient/family history. Some felt mistakes reflected the rushed nature of visits, the computer's demand for the clinician's attention, use of templates, and delays in writing notes. They expressed appreciation for access to notes and being able to identify mistakes. Some suggested new portal functionality that would allow patients to edit or point out needed corrections. They perceived a negative tone if they

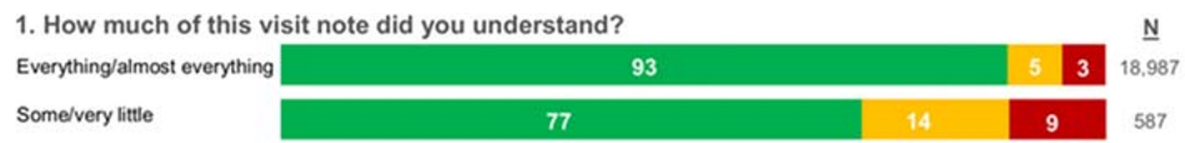

2. The visit note accurately described the visit. *

\begin{tabular}{l|c|c|c|c|}
\cline { 2 - 4 } Agree/somewhat agree & 95 & 3 & 18.530 \\
\cline { 2 - 4 } Disagree/somewhat disagree & & 95 & 10 & 882
\end{tabular}

3. Was there anything important to you that was missing in this visit note? *

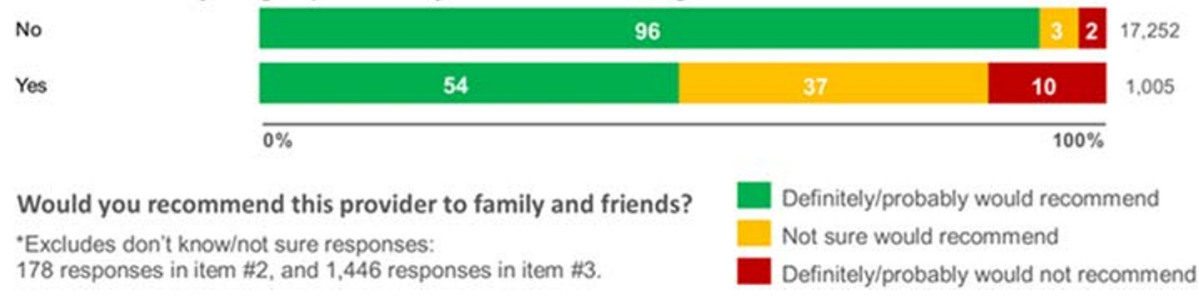

Figure 1 Willingness to recommend provider related to perceptions of visit note. 
Table 3 Examples from 2376 Patient Responses According to 9 Themes: "Do you have suggestions about how this visit note could have been better?"

Structure and content $(N=582$ responses $)$

It would be helpful to have a patient action plan for the next $\mathrm{x}$ months.

Keep the information in the note pertinent to that day's visit at the top, before past information.

... less "copying and pasting" of prior notes.

She gave me names as an informal referral, I lost the paper and read my notes trying to find it ... this type of info should be on top of the note, the first thing listed.

... the note felt like rather generic, as though it could have been written about another patient

Medical jargon $(N=524)$

It used medical terms that I didn't completely understand, but I was able to figure most of it out by doing an internet search.

Add a feature in the software to expand the short hand the doctor's use in their description of medical terms. DO NOT have the doctor's take any more time to write more than they have to.

Accuracy $(N=429)$

Seeing errors in the notes made me realize there had been a

miscommunication about a couple of things, which I could then clarify.

The information written about me personally was overtly wrong, i.e. mixing past circumstances with present situation.

The note had a different diagnose than the one I was told during the office visit.

Process $(N=182)$

... it was the apparent lack of coordination between providers (like they had not read another provider's note), I was able to inquire about it when I saw my provider.

I wish the wait time to read the note was shorter. I had to wait more than two weeks to read it.

She spent all her time typing (I'm sure it was her notes) and very little time talking to me.

Patient portal $(N=146)$

Notes should be an option on the first list on [portal]. They're too difficult to find!

The graphic design of the site and the notes could be improved. Not much visual hierarchy in the information.

Well I do see up to 3 different doctors- I believe it's important if there was a way to send the one doctor my concerns or issues and be able to copy the other two ...

Negative tone $(N=138)$

I expressed my concern over a symptom that I have been experiencing every day and felt was connected to my condition. The note dismissed it as an "occasional" occurrence and suggested that if it became bothersome I should see a different specialist. I wish the doctor had not brushed it off so quickly.

It should reflect what we both thought. Not just what the doctor thought. Especially if we disagree for the reason of symptoms.

Patient input $(N=97)$

It is best if doctors review the notes with patients before patient leaves. This ensure accuracy and concurrence ... thus reinforcing discussion and/or action plan.

There are often small inaccuracies. I am VERY frustrated that I have no easy way for sending in corrections.

It would be helpful to have a feedback mechanism.

Completeness $(N=54)$

Provider wrote that we discussed things we didn't, and missed some of the things we did discuss.

My Dr. can be too brief with his notes. Sometimes I have to ask him to elaborate.

Communication during the visit $(N=52)$

The visit note made it very apparent that the doctor never listened to my issues, and just made unilateral decisions about my care. More compassion and understanding on the part of the provider and a willingness to accept my responses without exhibiting frustration over her inability to book the surgical procedure.

Categories are not mutually exclusive; each comment could be assigned up to 3 codes

felt their concerns were unjustifiably dismissed, their perspectives not represented, or read terms such as "patient denies" and "obese."
They had ideas for improving patient portals. Many cited multiple steps required to find notes and suggested they be prominent on the homepage or visit summary. They suggested redesigning and updating the entire portal, improving mobile functionality, and adding print capability. Some commented on larger issues that might be addressed with new functions in electronic records, such as struggling with decentralization of their information when they see multiple providers, and expressing different, patient-specific needs for privacy (e.g., not wanting all providers to see the entire record, or not needing a secure login to read notes). Some requested avenues for patient input during or after the visit, using portal feedback mechanisms for comments.

Finally, patients expressed difficulties with various aspects of process and communication during visits. Some felt notes were not posted quickly enough following visits. Some described feeling rushed during visits; lack of attentiveness by clinicians busily typing during the encounter; and suboptimal follow-up, referrals, and communication among their clinicians.

An additional 1133 comments coded as "other" consisted almost entirely of praise for open notes $(n=812)$ and for clinicians or visit experiences $(n=316)$.

\section{DISCUSSION}

In this study, patients overall give high marks both to open notes and to those preparing them. Nearly all reported understanding everything or almost everything in a clinician's visit note. Contrary to our expectations, we found only a small difference in the proportions of patients who understood notes written by a primary care clinician or a specialist, and almost no differences according to the type of health professional (MD, NP, or PA). The patients' reports also pointed to how resourceful they are in finding answers to questions arising from notes without calling on their clinicians for explanations. Practitioners concerned that patients will not understand notes and will contact their practices for clarifications ${ }^{14}$ should find these results reassuring. They may serve also as a partial explanation for a phenomenon experienced almost invariably by practices implementing open notes: While patients report striking benefits, clinicians experience little perturbation in their practice and workflow.

In earlier work on patient-reported benefits of note reading, we found that Blacks and Hispanics, older patients, and those with fewer years of education were more likely to report benefits from note reading than others, and that non-English speakers and those with less education reported greater benefits to medication management. ${ }^{5,6}$ In the current study, we found that patients understood their notes regardless of their age, race, or socioeconomic group; there was a slight dip in understanding among those with a high school education or less which was consistent, though modestly so, with our hypothesis. In the information era, people are becoming used to having vast amounts of information at their fingertips and 
providing full transparency with online health records has come of age. All patients stand to gain from greater access to information about their health and care.

On the other hand, some patients wrote that notes made them feel their clinicians did not care about them or were too busy to provide individualized care. Although affecting only a relatively small proportion of patients, we also found that those reporting they understood only some or very little of the note, disagreed that the note accurately described the visit, or found something important missing may have trust affected substantially, as another study has suggested. ${ }^{17}$ However, we found fewer patients than hypothesized finding errors or omissions in their notes. While clinicians may not consider some of the reported errors clinically important, for patients they may suggest lack of attentiveness. The written comments suggest that accuracy is important to patients, whether referring to incorrect or to missing information, and that they would like a mechanism on the patient portal to offer corrections. This issue is complex, as evidenced by the many patients who would still recommend their providers despite noting errors. Nevertheless, consistent with our hypothesis, the likelihood of recommending a clinician to others was substantially lower when patients found a note inaccurate or incomplete.

Only about 1 in 5 respondents offered suggestions for improvement, but we should not assume the majority were satisfied. Those who did not write a response may have had limited expectations about their notes, or few ideas on how to improve them. Some respondents hesitated in their comments, reporting that they feared being labeled as a "difficult" or "problem" patient. Such reluctance has particular relevance for those working to reduce medical errors and increase diagnostic accuracy by calling on patients who read their notes. ${ }^{10}$ As patients become more familiar with reviewing their records on portals, devising user-friendly and secure methods for reporting and correcting errors will become more urgent.

As the practice of opening notes spreads, the commentary exampled in Table 3 provides further insights and points toward new initiatives. Perhaps most importantly, many patients suggested that notes should prominently and clearly indicate next steps in their treatment plans and offer explicit advice about what they should do to maintain health or better manage illness. This may point to a need to reorganize the structure and content of notes to maximize their usefulness for patients and providers. Greater attention to the treatment plan and goals of treatment could improve patient adherence and enhance ongoing evaluation of the care plan. In addition, many patients suggested minimizing abbreviations and what they viewed as medical jargon.

Some of the patients' suggestions for improving notes point to technical solutions, such as "mouse overs" that define terms and spell out acronyms, or provide links to glossaries. Voice recognition and other advancing technologies may improve accuracy and reduce typing during visits. While decisions about changes to the order of notes would be made by clinicians, making notes easier to find and making portals more patient friendly can best be addressed by those who design and build patient portals, working in collaboration with patients. ${ }^{18}$ These issues gain urgency at a time when patients' connections to their health information is accelerating. Under the $21 \mathrm{st}$ Century Cures Act, rules taking effect November 2, 2020 require patients be provided access to all the health information in their electronic medical records, with few exceptions. ${ }^{19}$ Clinical notes are included in the information that must be made available.

This study has important limitations. Patients may have selected those notes they most liked or best understood, not wanting to report on those they did not understand or could only dimly recall. Reporting that they understood the note might indicate they felt they understood what they needed to understand, rather than knowledge of the medical details. ${ }^{20}$ The survey solicited self-reports. It did not include efforts to confirm either patients' understanding or their reports of inaccurate or missing information, and future studies should solicit patient responses to notes selected at random from their records. Moreover, respondents were predominantly white and college educated, and our findings may not reflect the experiences of more diverse and less-educated patients; when patients at a safety-net hospital were handed the last note from their clinical record, several reported difficulties understanding the note. ${ }^{21}$ Further research among more diverse patient groups is needed to better understand the needs and suggestions from more disadvantaged patient populations. Finally, while it addressed a topic relevant to patients and used reminders and incentives, the survey's response rate was low, although not unexpectedly so for an online survey. ${ }^{4,22}$ When we compared respondents to non-respondents, we found modest differences between groups in that those responding to our survey had a heavier burden of major chronic conditions. In a previous report, we noted survey respondents were somewhat older than non-respondents. ${ }^{5}$ It may be fair to infer that patients who responded were those most interested in having access to their notes and, in turn, given their higher morbidity, may have the most to gain from reading them.

Spurred in part by the OpenNotes movement that began 10 years ago with a study of a few thousand patients, ${ }^{4}$ more than 50 million Americans today have ready access to their notes. ${ }^{3}$ The good news from this study is that those written by a broad array of clinicians were comprehensible to an equally broad range of readers, and that most patients found them accurately describing their visits. With no evidence of major disparities among patients according to education or other sociodemographic or health characteristics, these findings complement mounting evidence that open notes can empower patients to become more informed and engaged in their care. Informed patients, working in partnership with their clinicians, may both be safer and make better choices. Patients, clinicians, and health systems working to optimize the structure and quality of notes have a rich opportunity to inform, to generate mutual trust, and to improve the quality of care. 
Acknowledgments: The authors thank John Santa, MD, for his valuable suggestions and critique.

Corresponding Author: Jan Walker, RN, MBA; Division of General Medicine, Beth Israel Deaconess Medical Center, Boston, MA, USA (e-mail: Jwalker1@bidmc.harvard.edu).

Data Availability: The datasets generated during and/or analyzed during the current study are not available.

\section{Compliance with Ethical Standards:}

Conflict of Interest: The authors report funding from the Robert Wood Johnson Foundation, Gordon and Betty Moore Foundation, Peterson Center on Healthcare, and Cambia Health Foundation. The funders had no role in designing or conducting the study, analyzing the data, preparing the manuscript, or deciding to submit the manuscript for publication. Dr. Bell reports unrelated grant funding from the Agency for Healthcare Research and Quality within the past 36 months.

\section{REFERENCES}

1. Greene J, Hibbard JH. Why does patient activation matter? An examination of the relationships between patient activation and healthrelated outcomes. J Gen Intern Med. 2012;27(5):520-526. https://doi. org/10.1007/s11606-011-1931-2

2. Hibbard JH. Patient activation and the use of information to support informed health decisions. Patient Educ Couns. 2017;100(1):5-7. https:// doi.org/10.1016/j.pec.2016.07.006

3. OpenNotes ${ }^{\circledR}$. OpenNotes Homepage. OpenNotes. https://www. opennotes.org/. Accessed November 26, 2019

4. Delbanco $\mathbf{T}$, Walker $\mathbf{J}$, Bell SK, et al. Inviting patients to read their doctors' notes: A quasi-experimental study and a look ahead. Ann Intern Med. 2012;157(7):461-470. https://doi.org/10.7326/0003-4819-157-7201210020-00002

5. Walker J, Leveille S, Bell S, et al. OpenNotes After 7 Years: Patient Experiences With Ongoing Access to Their Clinicians' Outpatient Visit Notes. JMIR. 2019;21(5):e13876. https://doi.org/10.2196/13876

6. DesRoches CM, Bell SK, Dong Z, et al. Patients Managing Medications and Reading Their Visit Notes: A Survey of OpenNotes Participants. Ann Intern Med. May 2019. https://doi.org/10.7326/M18-3197

7. Mishra V, Hoyt R, Wolver S, Yoshihashi A, Banas C. Qualitative and Quantitative Analysis of Patients' Perceptions of the Patient Portal Experience with OpenNotes. Appl Clin Inform. 2019;10(01):010-018. https://doi.org/10.1055/s-0038-1676588

8. Nazi KM, Turvey CL, Klein DM, Hogan TP, Woods SS. VA OpenNotes: exploring the experiences of early patient adopters with access to clinical notes. J Am Med Informatics Assoc. 2015;22(2):380-389. https://doi.org/ 10.1136/amiajnl-2014-003144

9. Jackson SL, DesRoches CM, Frosch DL, Peacock S, Oster N V., Elmore JG. Will use of patient portals help to educate and communicate with patients with diabetes? Patient Educ Couns. 2018;101(5):956-959. https://doi.org/10.1016/j.pec.2017.11.004

10. Bell SK, Mejilla R, Anselmo M, et al. When doctors share visit notes with patients: a study of patient and doctor perceptions of documentation errors, safety opportunities and the patient-doctor relationship. BMJ Qual Saf. 2017;26:1-9. https://doi.org/10.1136/bmjqs-2015-004697

11. Fan W, Yan $\mathbf{Z}$. Factors affecting response rates of the web survey: A systematic review. Comput Human Behav. 2009;26: 132-139. https://doi. org/10.1016/j.chb.2009.10.015

12. Glasheen W, Cordier T, Gumpina R, Haugh G, Davis J, Renda A. Charlson Comborbidity Index: ICD-9 Update and ICD-10 Translation. Vol 12.; 2019.

13. Benjamin EJ, Paul Muntner C, Chair Alvaro Alonso V, et al. Heart Disease and Stroke Statistics 2019 Update: A Report From the American Heart Association. Circulation. 2019;139:56-528. https://doi.org/10. 1161/CIR.0000000000000659

14. Chen G, Khan N, Walker $\mathbf{R}$, Quan $\mathbf{H}$. Validating ICD coding algorithms for diabetes mellitus from administrative data. Diabetes Res Clin Pract. 2010;89(2): 189-195. https://doi.org/10.1016/j.diabres.2010.03.007

15. Braun V, Clarke V. Thematic Analysis. In: Cooper H, ed. APA Handbook of Research Methods in Psychology. American Psychological Association; 2012.

16. L. Fleiss J. Statistical Methods for Rates and Proportions. 2nd ed. New York, NY: John Wiley \& Sons, Ltd; 1981.

17. Platonova EA, Kennedy KN, Shewchuk RM. Understanding Patient Satisfaction, Trust, and Loyalty to Primary Care Physicians. Med Care Res Rev. 2008;65:696-712. https://doi.org/10.1177/ 1077558708322863

18. Irizarry T, DeVito Dabbs A, Curran CR. Patient Portals and Patient Engagement: A State of the Science Review. J Med Internet Res. 2015;17(6):e148. https://doi.org/10.2196/jmir.4255

19. U.S. Department of Health and Human Services. 21st Century Cures Act: Interoperability, Information Blocking, and the ONC Health IT Certification Program.; 2019. https://www.govinfo.gov/content/pkg/FR-2019-03-04/ pdf/2019-02224.pdf.

20. Huang JS, Yueh R, Ma S, Cruz R, Bauman L, Choi LJ. Adolescents' and Young Adults' Satisfaction with and Understanding of Medical Notes from a Pediatric Gastroenterology Practice: A Cross-Sectional Cohort Study. J Pediatr. 2019;215:264-266. https://doi.org/10.1016/j.jpeds.2019.06.052

21. Belyeu BM, Klein JW, Reisch LM, et al. Patients' perceptions of their doctors' notes and after-visit summaries: A mixed methods study of patients at safety-net clinics. Heal Expect. 2018;21(2):485-493. https:// doi.org/10.1111/hex. 12641

22. NORC. Methodology Report: 2014-2015 Nationwide CAHPS Surveys of Adults Enrolled in Medicaid between October and December 2013.; 2015. https://www.medicaid.gov/medicaid/quality-of-care/downloads/performance-measurement/methodology-report.pdf.

Publisher's Note Springer Nature remains neutral with regard to jurisdictional claims in published maps and institutional affiliations. 